\title{
Implementation Intentions Facilitate Response Inhibition in Children with ADHD
}

\author{
Caterina Gawrilow $\cdot$ Peter M. Gollwitzer
}

\begin{abstract}
Attention Deficit/Hyperactivity Disorder (ADHD) is associated with action control problems such as failure to inhibit inappropriate responses. Two studies investigated whether self-regulation by implementation intentions (if-then plans; Gollwitzer, P. M. (1999). Implementation intentions: Strong effects of simple plans. American Psychologist, 54, 493-503) facilitates response inhibition in children with ADHD. In Study 1, children with ADHD who furnished a suppression goal with implementation intentions improved inhibition of an unwanted response on a Go/NoGo task to the same level observed in children without ADHD. Study 2 showed that a combination of implementation intentions and psychostimulant medication resulted in the highest level of suppression performance in children with ADHD. Theoretical and applied implications of these results are discussed.
\end{abstract}

Keywords Children with ADHD - Executive functions ·

Response inhibition · Self-regulation · Implementation intentions

C. Gawrilow $(\square)$

University of Konstanz, Box D 39, Konstanz 78457, Germany

e-mail: caterina.gawrilow@uni-konstanz.de

P. M. Gollwitzer

Psychology Department, New York University, 6 Washington Place, New York, NY 10012, USA

e-mail: peter.gollwitzer@nyu.edu 


\section{Introduction}

Impulsivity is one of the defining symptoms of children with Attention Deficit/ Hyperactivity Disorder (ADHD). ${ }^{1}$ Children with ADHD not only demonstrate a deficient inhibitory control in their everyday behavior, but also impaired performance on different tasks measuring inhibitory control, such as the Continuous Performance Task (CPT, Rosvold, Mirsky, Saranson, Bransome, \& Beck, 1956) and the Stop Signal Task (SST, Logan \& Cowan, 1984). Children with ADHD tend to make more errors of false rejection (i.e., not pressing a key when they are supposed to do so) and false alarm (i.e., pressing a key when they are not supposed to do so) than children without ADHD (Nichols \& Waschbusch, 2004). Several other studies (e.g., Oosterlaan \& Sergeant, 1996; Pliszka, Borcherding, Spratley, Leon, \& Irick, 1997) have found that children with ADHD have slower stop signal reaction times than healthy comparison children on the SST (Oosterlaan, Logan, \& Sergeant, 1998; Smith \& Taylor, 2006). Recent studies assessing brain activity support these findings in suggesting that children with ADHD demonstrate specific impediments in the reaction to No-Go trials or stop signals compared to children without ADHD (e.g., Overtoom et al., 2002; Pliszka et al., 2006; Smith, Taylor, Brammer, Toone, \& Rubia, 2006).

There is current debate as to how these group differences are interpreted best (Miyake, Friedman, Rettinger, Shah, \& Hegarty, 2001; Zelazo, Qu, \& Müller, 2005). One explanation is that poor response inhibition constitutes the core executive deficit in childhood ADHD (Barkley, 1997; Tannock, 1998), while another approach considers deficits in working memory to be central to the executive dysfunction associated with ADHD (Pennington, Bennetto, McAleer, \& Roberts, 1996; Willcutt, Pennington, Olson, Chhabildas, \& Hulslander, 2005). Furthermore, whereas some researchers point to a weakened general executive functioning in children with ADHD (Scheres et al., 2004), others argue that more specific deficits of action control are to blame (see, e.g., the delay aversion theory of ADHD proposed by Sonuga-Barke, 2002). Indeed, the research on executive function deficits in children with ADHD has produced a somewhat incoherent picture, with some studies failing to find differences on several measures of executive functioning and other studies only allowing vague inferences regarding the exact nature of the executive dysfunction observed (Halperin \& Schulz, 2006; Wu, Anderson, \& Castiello, 2006). Still, even though weaknesses in executive functioning seem to be neither necessary nor sufficient to cause all cases of ADHD, it seems adequate to conclude that individuals with ADHD are at a higher risk for performing poorly on tasks that require effective behavioral inhibition.

Despite recent progress in cognitive neuro-psychology, researchers nevertheless assert that "the field still lacks a compelling theory of executive functions" (Miyake, Friedman, Emerson, Witzki, \& Howerter, 2000; p. 50). Commonly, however, and most relevant to the present research, executive functions are considered to be "general-purpose control mechanisms that modulate the operation of various cognitive sub-processes and thereby regulate the dynamics of human cognition" (Miyake et al., p. 50). Executive functions thus describe a group of cognitive processes that enable a person to employ successful action control. The neural substrates of executive functions are housed in areas of the

\footnotetext{
${ }^{1}$ For ease of exposition in this paper, the term ADHD is used constantly as an abbreviation for Attention-Deficit/Hyperactivity Disorder according to the DSM-IV subtypes of ADHD (Predominantly Inattentive Type, Combined Type, Predominantly Hyperactive-Impulsive Type) and the ICD-10 category of Hyperkinetic Disorder.
} 
frontal lobes, more precisely the prefrontal cortex (Zhu, 2004). Thus, disorders resulting in faulty action control (e.g., patients with frontal lesions) inform us about the consequences of (dysfunctional) executive functions (Posner \& Rothbart, 2000).

\section{Self-regulation by implementation intentions}

The present research explores whether children with ADHD can compensate for their deficits in executive functioning through the use of self-regulation strategies. More specifically, this research aims to determine whether the use of certain self-regulation strategies alleviates performance deficits in tasks requiring effective response inhibition. A meta-analysis by Reid and colleagues reports that self-regulation interventions in children with ADHD can indeed improve performance on tasks that require executive control (Reid, Trout, \& Schartz, 2005). Specifically, the authors explored selfmonitoring and self-management strategies. They define self-monitoring as the process by which individuals carefully scrutinize and document their own behavior first, through discriminating a target response and second, through self-recording "some dimension of the target response" (Reid et al., 2005, p. 362). For instance, a child working on a spelling task is required to observe her or his performance and record the amount of task completion. Self-management demands rating and comparing some aspect of a behavior to an external standard or criterion. For instance, a child working on a spelling task is required to observe and evaluate her or his performance. Hence, self-monitoring and self-management methods are relatively general strategies of action control, serving to improve task performance through observation of one's behavior and self-evaluation.

A powerful self-regulation strategy geared towards facilitating the control of specific critical goal-directed behaviors is the making of if-then plans (i.e., forming implementation intentions, Gollwitzer, 1999). Self-regulation by implementation intentions entails delegating action control to pre-specified critical environmental cues. In other words, by planning out in advance when, where, and how a goal is to be transformed into action, implementation intentions disencumber executive functions. As a result, deficits in executive functioning should no longer be apparent in the quality of task performance. Implementation intentions are expressed as "If situation $\mathrm{X}$ is encountered, then I will perform the goal-directed behavior Y". Essentially these statements link a critical anticipated situation (if-part) with a selected goal-directed behavior (then-part). Furthermore, implementation intentions need to be distinguished from goal intentions. Whereas goal intentions specify preferred end-states that the individual feels committed to achieve, implementation intentions spell out how the goal intention is to be realized.

Gollwitzer $(1993,1996)$ encapsulates the function of implementation intentions in the metaphor "passing the control of one's behavior on to the environment" (1993, p. 173). By forming implementation intentions, people can strategically turn effortful control of their goal-directed behaviors into direct control by specified situational cues. The effects of implementation intentions are due to cognitive processes pertaining to both the specified critical situation (if-part) and the intended goal-oriented behavior (then-part). First, the mental representation of the critical situation becomes activated and therefore highly accessible. This accessibility allows for easy detection, effective recall, and a readiness to attend to the critical situation, even if one is cognitively busy (e.g., Aarts \& Dijksterhuis, 2000; Parks-Stamm, Gollwitzer, \& Oettingen, 2007; Webb \& Sheeran, 2003). Second, the initiation of the planned goal-directed behavior acquires features of automaticity: in the presence of the specified situation, action initiation is immediate, 
efficient, and no longer requires conscious intent (e.g., Brandstaetter, Lengfelder, \& Gollwitzer, 2001; Sheeran, Webb, \& Gollwitzer, 2005; Webb \& Sheeran, 2007).

As recently reported in a meta-analysis, implementation intentions facilitate the attainment of all kinds of goals (e.g., academic, health, and interpersonal goals; Gollwitzer \& Sheeran, 2006). In particular, implementation intentions appear to support the attainment of difficult goals. For instance, research shows that goal attainment is facilitated when goals have to be acted on at inconvenient times (Gollwitzer \& Brandstaetter, 1997), when they are somewhat unpleasant to perform (Orbell, Hodginks, \& Sheeran, 1997), and when they are easily forgotten (Sheeran \& Orbell, 1999). Supplementing these goals with implementation intentions leads to a higher goal attainment rate. Furthermore, implementation intentions ease the control of unwanted influences on an ongoing goal pursuit. They shield the focal goal striving from getting derailed from internal (e.g., bad mood) and external (e.g., temptations) disruptions, thus allowing for successful goal realization, even in the face of hindrances (Gollwitzer, Bayer, \& McCulloch, 2005).

Most relevant to the present research, implementation intentions were also found to support performance on two tasks where challenges to executive control are especially prevalent, namely task switching and spatial Simon task paradigms (Cohen, Bayer, Jaudas, \& Gollwitzer, in press). In task switch paradigms executive control is necessary to avoid task-switching costs; in the Simon task, executive control is necessary to minimize the influence of spatial location on stimulus classification. Implementation intentions prove effective for these types of tasks for two reasons: (a) they heighten activation of the mental representation of critical stimuli, and (b) they strongly link critical stimuli to the appropriate goal-directed behavior. Thus, forming implementation intentions in the context of task-switch paradigms as well as Simon tasks improves performance (i.e., reduces task switching costs and the influence of spatial location, respectively). Apparently, the strategic automaticity (i.e., direct stimulus control) produced by implementation intentions minimizes the demands of these tasks on executive functioning and thus improves performance. In line with this interpretation, research also demonstrates that implementation intentions are helpful for people facing chronic action regulation problems. For instance, patients with frontal brain lesions (Lengfelder \& Gollwitzer, 2001), schizophrenic inpatients, and opiate addicts under withdrawal (Brandtstaetter et al., 2001, Studies 1 and 2) have all been shown to benefit from using implementation intentions; these positive effects of implementation intentions prevail even under conditions that make effective action control especially challenging (e.g., high-cognitive load).

\section{The present research}

Given that children with ADHD also show severe action control problems, as evidenced in their weak performances on executive function tasks, we hypothesized that forming implementation intentions (Gollwitzer, 1999) should diminish executive demands and thus help these children perform better on such tasks. Because children with ADHD show the most prominent deficits in executive functions tasks requiring the inhibition of an inappropriate response (e.g., Booth et al., 2005; Fischer, Barkley, Smallish, \& Fletcher, 2005; Oosterlaan et al., 1998; Pliszka, Liotti, \& Woldorff, 2000), we focused on response inhibition to test our hypothesis. 
In past research, two different tasks have been used to assess the inhibitory ability of children with ADHD, namely the CPT (Rosvold et al., 1956), and the SST (Logan \& Cowan, 1984). For the two studies reported in this paper, we developed a new task paradigm (i.e., a classification task combined with a Go/No-Go task) modeled after both the CPT and the SST. Specifically, five different means of transportation (plane, car, truck, ship, and train) and five different animals (chicken, cat, mouse, cow, and pig) were presented on a computer screen. The task called for participants to both classify stimuli by pressing a particular computer key (classification task, Go trials), as well as inhibit classification in response to a stop signal $(200-\mathrm{Hz}$ sound). The stop signal appeared on one third of the 300 trials, $150 \mathrm{~ms}$ before the stimulus (No-Go, stop trials). The computer key assignment was switched during the experiment in order to make the task more difficult and to counterbalance the keys.

In both experiments, we randomly assigned children with ADHD to one of two groups: Children in the goal intention group formed a goal to inhibit a classification response for marked stimuli, while children in the implementation intention group, in addition to forming a goal intention, formed an if-then plan. Furthermore, we decided against a control group for this study (i.e., participants with neither a goal intention nor an implementation intention) based on research showing that individuals provided with mere task instructions (i.e., standard control condition) perform the same as individuals with goal intentions (Cohen et al., in press; Gollwitzer \& Sheeran, 2006).

We measured both the number of successful stops on the No-Go trials and the response times for the Go trials. In Study 1 we compared the performances of children with and without ADHD, while in Study 2 we compared the performances of children with ADHD with and without psychostimulant medication. We hypothesized that children with ADHD, in general, should show lower levels of inhibition performance than children without ADHD, and that implementation intentions should facilitate the ability to inhibit automatic responses. We expected the benefits of implementation intentions to be particularly strong for children with ADHD taking prescription medication.

\section{Study 1: the effect of implementation intentions on inhibition performance in children with ADHD}

\section{Overview}

In Study 1, we hypothesized that children with ADHD, in comparison to children without ADHD, should demonstrate poorer inhibition on the Go/No-Go task and should respond more slowly on the classification task. We also expected children in the implementation intention condition (i.e., children with both a goal- and implementation intention) to show improved inhibition of the critical response. That is, these children should not respond to critical stimuli (i.e., pictures that were accompanied by a sound). Furthermore, based on the finding that implementation intention effects are greater for tasks with high difficulty (Gollwitzer \& Brandstaetter, 1997), we predicted that children with ADHD, in contrast to children without psychological disorders, should profit more from forming implementation intentions since the task should be more difficult for them. Finally, we expected that the use of implementation intentions should have no costs (i.e., classification response times should not be slowed) as action control by 
implementation intentions is efficient (i.e., requires few cognitive resources; Brandstaetter et al., 2001).

Method

\section{Participants}

Because the prevalence of ADHD is more frequent in males (Barkley, 1990), we only asked male children to participate in our study. Fifty-eight male children with ages ranging from 8.4 to 14.1 years $(M=11.2 ; S D=1.3)$ participated in this experimental study after giving informed consent. The study was approved by the ethics committee of the University of Konstanz. The clinical group, consisting of 30 boys with ADHD, was recruited from a collaborating local pediatric clinic (SPZ, Sozialpaediatrisches Zentrum Konstanz, Germany). These children, diagnosed with Hyperkinetic Disorder F90.0 (ICD-10; World Health Organization (WHO), 1991) as their primary disorder, were selected by the head child psychiatrist at the clinic to participate in our study. To recruit the nonclinical comparison children $(n=28)$, we sent invitation letters to parents in the community who had male children in the same age and in the same type of schools as the ADHD sample. All parents were paid ( $€ 10$, approximately 12 USD) for their children's participation.

With regard to ethnic background, all of the ADHD and all of the control boys were Caucasian representing the very homogenous population of the city of Konstanz/ Germany. A socioeconomic status index (SES) was obtained based on the parents' educational level and occupation. There were no significant differences between children with and without ADHD concerning socioeconomic status (Table 1).

To assess differences between children with and without ADHD and confirm the diagnosis, we asked the parents of all participating children to fill out two questionnaires: the CBCL (Child Behavior Check List, Arbeitsgruppe Deutsche Child Behavior Checklist, 1998) to measure different aspects of their children's behavior (e.g., social withdrawal, somatic disturbances, and anxiety/depression in the internal scale; antisocial and aggressive behavior in the external scale; social problems, schizoid/obsessive compulsive behavior and attention problems plus the aforementioned internal and external scales in the total CBCL) and a questionnaire assessing the current medication status of their children. Table 1 presents the parents' evaluations from the CBCL according to the main scales. The CBCL showed significant differences between the ADHD group and the control group, total CBCL $t(57)=7.14, p<.001$, internal CBCL $t(57)=3.71, p<.001$, external CBCL $t(57)=5.52, p<.001$. Within the ADHD group,

Table 1 Background variables of the sample of Study 1

\begin{tabular}{|c|c|c|c|c|c|c|}
\hline \multirow[t]{2}{*}{ Background variables } & \multicolumn{4}{|c|}{ Children } & \multirow[t]{2}{*}{$t$} & \multirow[t]{2}{*}{$p$} \\
\hline & ADHD & & Contro & & & \\
\hline SES & 2.20 & $(.64)$ & 2.32 & $(.52)$ & .78 & .434 \\
\hline CBCL Internal & 63.67 & $(13.52)$ & 52.44 & $(8.55)$ & 3.71 & .001 \\
\hline CBCL External & 67.41 & $(9.21)$ & 53.25 & $(10.30)$ & 5.52 & .001 \\
\hline Total CBCL & 69.19 & (7.61) & 53.77 & $(8.81)$ & 7.14 & .001 \\
\hline
\end{tabular}

Note. Standard deviations in parentheses 
13 children were medicated with one form of methylphenidate (MPH), whereas the remaining 17 children were not medicated with MPH at the time of investigation.

\section{Design}

The experiment followed a 2 between (Group: ADHD versus no ADHD) $\times 2$ between (Intention: goal intention versus implementation intention) $\times 2$ within (Blocks: $1-5$ vs. 6-10) factorial design. The main dependent variable was the mean frequency of response inhibition (i.e., how often the participants inhibited responding when the stop signal occurred expressed as a percentage). Furthermore, we assessed the mean classification response times.

\section{Material}

The stimuli were presented on a 15" computer screen. They were viewed at a distance of approximately $45 \mathrm{~cm}$ (17.71 in). The discrimination task stimuli contained pictures of animals (chicken, cat, mouse, cow, and pig) and means of transportation (plane, car, truck, ship, and train). All images were approximately the same size, covered a maximum visual field of $7.78^{\circ}$, and were painted by a professional artist. Each stimulus was preceded by a 500-ms fixation cross, presented in the center of the screen. Either an animal or a means of transportation was presented in the centre of the screen for $1,000 \mathrm{~ms}$. Thereafter, a blank screen appeared for 1,500 ms. Participants had to classify the presented stimuli as either animals or means of transportation by pressing one of two marked keys on the keyboard with the index fingers of their left or right hand, respectively. One-third of the time, stop signals were presented $150 \mathrm{~ms}$ before the appearance of the stimulus. Figure 1 illustrates an example of both a Go trial and a NoGo trial.

\section{Procedure}

Computerized task. Participants completed the Go/No-Go task in a single session lasting approximately $20 \mathrm{~min}$. The paradigm involved two concurrent tasks. The Go trials required the classification of animals and means of transportation. In the No-Go trials, the presentation of a stop signal informed the participants to inhibit their classification response on that particular trial.

Informed consent was obtained from the participants' parents stressing that children or the parent(s) could withdraw their consent at any time with no unfavorable consequences. During task instructions the children faced the computer screen with the experimenter sitting next to them giving standardized verbal instructions. Furthermore, the experimenter was explicitly trained not to give more information than written on the instruction sheet. Children first performed a block of 30 practice trials. During this block, they practiced classifying the animals or the means of transportation by pressing the designated keys on the keyboard. We instructed participants to respond as quickly as possible without making any errors. In the second block of 30 practice trials, participants again were instructed to press an appropriate key to classify the pictures, but this time they were told to refrain from hitting any key when they heard a sound (i.e., the stop signal). During the performance of the task the experimenter sat quietly in a corner of the experimental cubicle. 

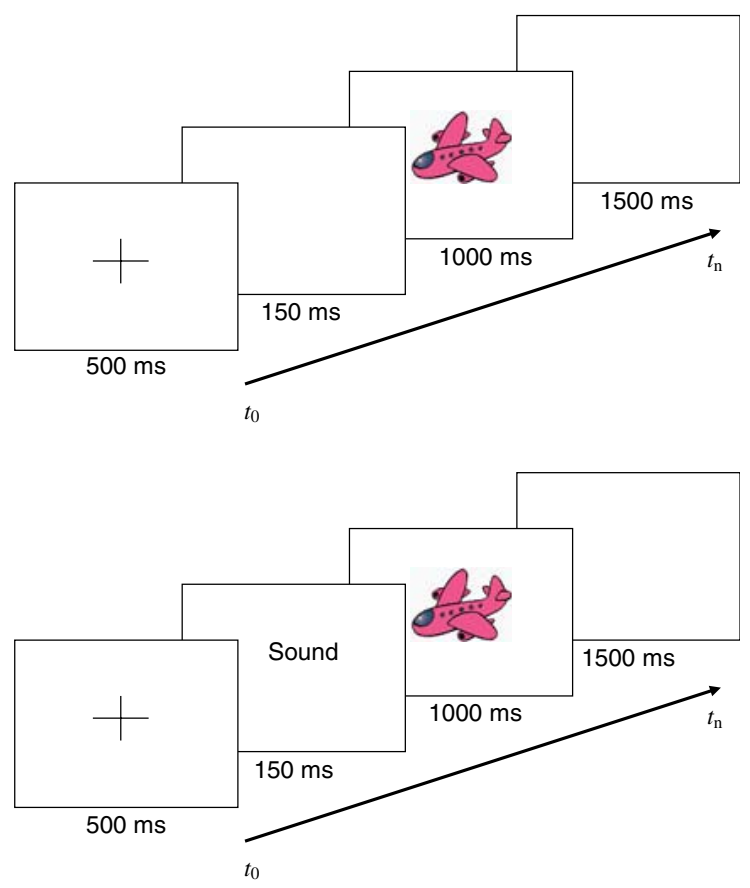

Fig. 1 Examples of a Go trial and a No-Go trial with respect to a transportation stimulus (Plane)

After the two practice blocks, participants completed five blocks of 30 trials. On onethird of the trials, that is on 50 trials (10 per block), participants heard the stop tone. At the end of the five blocks participants had a break, during which they were told that the designated keys for animals and means of transportation would be switched for the rest of the experiment. The final part of the experiment contained the same amount of training trials (30), training trials with stop signals (30), and again five experimental blocks of 30 trials each. Altogether, every child completed 10 blocks of 30 trials (300 trials in total), 100 of which were stop trials.

The stimuli were presented in random order for each participant. Throughout the experiment, there was an equal number of animals and means of transportation in each block. Thus, animals appeared in $50 \%$ of the trials, and each kind of animal was presented 30 times. The same was true for the various means of transportation.

Goal manipulation. The participants were randomly assigned to one of two intention conditions: goal intention versus implementation intention. Children in the goal intention condition set the goal to not press any key when a sound occurred together with the stimulus ("I will not press a key for pictures that have a sound!"). Children in the implementation intention condition, however, were asked to make an additional plan to the goal intention, "And if I hear a tone, then I will not press any key!" The experimenter asked the children in both conditions to repeat the self-regulatory instruction (goal intention or implementation intention) to themselves three times.

Interview. At the end of the experiment, the experimenter conducted a short interview with each participant. We asked children four general questions about how they experienced the computer game ("Did the instructions help you complete the task;" "Do you think that the game was easy;" "How much fun did you have while 
completing the task;" and "How many errors do you think you made.") Children answered these questions using a seven-point scale $(1=$ strongly agree, $7=$ strongly disagree). Finally, the children and their parents were thanked and extensively debriefed.

Results

\section{Response inhibition}

Table 2 illustrates the mean percentage of correct responses to stop signals in children with and without ADHD. A 2 (Intention: goal intention versus implementation intention $) \times 2$ (Group: ADHD versus no ADHD) $\times 2$ (Blocks: 1-5 versus 6-10) repeated measurement ANOVA on response inhibition was computed. There was no significant main effect of Block indicating that the response inhibition did not change over time. This analysis revealed a significant main effect of Intention, $F(1,54)=8.71$, $p<.01$, and a marginally significant main effect of Group, $F(1,54)=3.39, p=.07$. These main effects were qualified by a significant interaction effect, $F(1,54)=4.20$, $p<.05$, between the Intention and Group factors, indicating that the effect of forming implementation intentions was dependent on ADHD status.

An analysis of covariance was used to assess whether implementation intentions still lead to better response inhibition after controlling for the age of the children. Results indicated no significant effect of the covariate Age, $F(1,58)=.985, n s$, and the main and interaction effects of Intention and Group (reported above) did not change significantly.

Whereas children with ADHD who formed implementation intentions were better at inhibiting their responses $(M=89.90 \%, S D=5.84)$ than children with ADHD who formed only goal intentions $(M=78.26 \%, S D=9.65), t(28)=4.05, p<.001$, the children without ADHD did not show a significantly better response inhibition in the implementation intention condition $(M=89.44 \%, S D=7.09)$ than in the goal intention condition $(M=87.34 \%, S D=11.58), t(26)<1$, $n s$. Further $t$-tests revealed a significantly different response inhibition between children with and without ADHD in the goal intention condition, $t(28)=2.28, p<.05$, but not in the implementation intention condition, $t<1, n s$. Finally, no main or interaction effects of the Block factor (1-5 vs. $6-$ 10) were observed.

Table 2 Means and standard deviations for response inhibition and classification response times in children with and without ADHD by Intention Condition (Study 1)

\begin{tabular}{lll}
\hline Group & Intention condition & \\
\cline { 2 - 3 } & Goal intention & Implementation intention \\
\hline Children with ADHD & & \\
Response inhibition (\%) & $78.26(9.65)$ & $89.90(5.84)$ \\
Response time (ms) & $687.28(99.42)$ & $620.56(94.93)$ \\
Control children & & \\
Response inhibition (\%) & $87.34(11.58)$ & $89.44(7.09)$ \\
Response time (ms) & $620.81(81.01)$ & $597.96(96.92)$ \\
\hline
\end{tabular}

Note. Standard deviations in parentheses 


\section{Further analyses}

Response times. We found no difference in response times as a result of counterbalancing the keys midway through the experiment, $t(56)<1$, ns. Table 2 illustrates the mean response times (in ms) to the Go stimuli. A 2 (Intention: goal intention versus implementation intention) $\times 2$ (Group: ADHD versus no ADHD) ANOVA on response time was computed. The analysis revealed a marginally significant main effect of Group, $F(1,57)=3.30, p=.07$, and a marginally significant main effect of Intention, $F(1,57)=3.33, p=.07$. Children in the goal intention condition showed slower response times than children in the implementation intention condition, and children with ADHD demonstrated slower response times than control children (see Table 2). The interaction of the Group and Intention factors was not significant.

Individual differences. In order to check whether the beneficial effects of forming implementation intentions on response inhibition in children with ADHD are affected by the degree of behavioral disturbances attested to these children by their parents, we computed correlation coefficients between CBCL scores and stop performance. However, none of the three CBCL scales revealed a significant negative correlation coefficient: internal scale $r(14)=.00, n s$; external scale $r(14)=-.29, p>.25$; and total scale $r(14)=-.05, n s$. These nonsignificant correlation coefficients suggest that children with ADHD who suffered from pronounced behavioral disturbances did not benefit less from forming implementation intentions than children who were less burdened.

Interview. Overall, ANOVAs and two-tailed $t$-tests showed no significant differences between the two different group and intention conditions with respect to the following three questions asked during the children's interview: "Did the instruction help you complete the task;" "How much fun did you have while completing the task;" and "How many errors do you think you made." However, a two-tailed $t$-test, $t(57)=2.29$, $p<.05$, confirmed a difference between the ADHD and the no-ADHD control group concerning the question: "Do you think that the game was easy." Children with ADHD found the task easier $(M=2.64, S D=1.72)$ than did children without ADHD $(M=3.75, S D=1.06)$. This finding is in line with research demonstrating that children with ADHD often have distorted self-perceptions of competence (e.g., Owens \& Hoza, 2003).

\section{Discussion}

In the present study, children with ADHD benefited from forming implementation intentions during a stop task requiring the inhibition of cued actions. In particular, when given an implementation intention (as compared to a mere goal intention) the mean performance of children suffering from ADHD was significantly elevated to the performance level of the no-ADHD control children. The finding that children without ADHD did not profit from implementation intentions is consistent with research showing that the beneficial effects of implementation intentions are most apparent with (tasks) goals that are difficult to implement (Gollwitzer \& Brandstaetter, 1997). Indeed, the inhibition task seemed to be much easier for control children without ADHD as compared to children with ADHD (Pliszka et al., 1997), as the former showed an inhibition performance of close to $90 \%$ even without the formation of implementation intentions (the respective performance level of children with ADHD was a mere $78 \%$ ). 
Interestingly, children with ADHD who formed implementation intentions suffered no costs. In fact, there was a tendency for ADHD participants who formed implementation intentions to respond faster on the Go trials than ADHD participants with a goal intention. Apparently, children with ADHD could reap the benefits of implementation intentions on response inhibition without having to pay a price in terms of reduced classification performance. This finding supports the assumption (Gollwitzer, 1999) that action control by implementation intentions is an efficient strategy (i.e., requires few cognitive resources). Also, children with ADHD who had received weak behavior ratings by their parents (i.e., higher scores on the scales of the CBCL) benefited as much from forming implementation intentions as children with ADHD who had received better ratings. This suggests that delegating the control of behavior to external cues (in the present case, a stop behavior to the presence of a tone signal) frees action control from the chronic behavioral handicaps associated with the person (i.e., chronic shortcomings the person brings along into the critical situation; Gollwitzer et al., 2005).

In Study 2, we tried to remedy several shortcomings of Study 1. First, in Study 1 we did not assess how forming implementation intentions affected participants' commitment to do well on the task at hand. It seems plausible that the beneficial effects of implementation intentions observed in Study 1 are simply due to an increase in task commitment. To investigate this potential alternative explanation, in Study 2 we assessed participants' task commitment after manipulating participants' goal intention versus implementation intention. Goal commitment questionnaires have high reliability and validity (Seijts \& Latham, 2000). Thus, a straightforward goal commitment questionnaire allows assessing the degree to which the children actually want to act on the respective intention (i.e., goal or implementation intention).

Second, in Study 1 we did not vary the medication with MPH in children with ADHD. MPH reduces ADHD symptoms (Jensen et al., 2001) and has been found to increase inhibition abilities in children with ADHD (Bedard et al., 2003). The reason for this positive influence is that MPH, the agent in commonly prescribed psychostimulants, is a dopamine reuptake inhibitor. Hence, MPH enhances the availability of dopamine in the synaptic cleft that in turn increases the inhibition potential in areas of the prefrontal cortex. Research additionally shows that ADHD is associated with decreased activity in frontal brain areas and increased activity in posterior (parietal and occipital) brain areas (Durston, 2003), and that MPH affects not only frontal but also posterior areas (Mehta et al., 2000). Consequently, MPH is expected to positively influence inhibition responses and thus the execution of the behavior specified in the implementation intention in Study 1.

\section{Study 2: the effect of implementation intentions and methylphenidate on response inhibition in children with ADHD}

Overview

We invited only children with ADHD to participate in Study 2; those who participated were instructed not to take their MPH medication during the $48 \mathrm{~h}$ prior to testing. Children's inhibition performance was measured twice in the combined classification and Go/No-Go task used in Study 1. The children came to us in the morning after the 
48-h medication hiatus, and worked on this task. Thereafter, parents administered the children's usual medication and after a period of $60 \mathrm{~min}$ to allow the medication to take effect we reassessed the children on the same task. This procedure has been recently used by Hood, Baird, Rankin, and Isaacs (2005) demonstrating that children with ADHD show significant improvement in cognitive attention processes (i.e., tasks taken from the Test of Everyday Attention for Children TEA-Ch; Manly et al., 2001) after administration of MPH.

We expected that children with ADHD would benefit from forming implementation intentions and from medication with MPH. Furthermore, we predicted an interaction effect such that children with ADHD would profit most from a combination of implementation intentions and medication.

Method

\section{Participants}

Twenty boys with ADHD between the ages of 8 and 14 years old $(M=11.15$ years; $S D=1.65$ years) participated in this study. Again, in terms of ethnic background, all children were Caucasian. All children met ICD-10 criteria for Hyperkinetic Disorder (WHO, 1991), were diagnosed at a local pediatric clinic (SPZ, Germany) and received MPH medication ${ }^{2}$ (doses ranging between 15 and $20 \mathrm{mg}$ ). Only children who did not participate in Study 1 could take part in Study 2. The study was approved by the ethics committee of the University of Konstanz and is compliant with the World Medical Association declaration of Helsinki (The World Medical Association, 2004).

We sent a letter to numerous families with ADHD children taking stimulant medication. In this letter we informed parents, in detail, about the experimental procedure as well as the preconditions for participation. More specifically, we notified parents that for this study, their children should not receive medication for $48 \mathrm{~h}$ prior to the experiment, and due to possible interaction effects with the action of the medication (Midha, McKay, Rawson, Korchinski, \& Hubbard, 2001) no fatty meal should be eaten on the day of the experiment. A few days after having sent the letter, the experimenter called the parents to make sure that they had received the letter and to ask them about their willingness to participate. If parents gave consent for their children to participate in the study, a 2-h appointment was arranged. After completing the testing session, all children received a small bag of low calorie candy. In addition, participant's parents received $€ 15$ (approximately 18 USD) as reimbursement for their travel expenses plus a written report on their children's performance on the computerized test.

\section{Design}

The study followed a 2 between (Intention: goal intention versus implementation intention) $\times 2$ within (Medication: yes versus no) factorial design. We assigned children randomly to either the goal intention or the implementation intention condition. All children completed the entire task procedure twice (at first without MPH, then with $\mathrm{MPH}$ ). As dependent variables we measured the frequency of correct stop responses in the No-Go trials, and the response times in the Go trials.

\footnotetext{
$\overline{{ }^{2} \text { No titration algorithm was used. }}$
} 


\section{Material}

For this study we altered the computerized task from Study 1 (see Fig. 1) by omitting the counterbalancing of key assignment. Because Study 1 showed that classifying means of transportation or animals with left or right keys did not have an influence on the results, counterbalancing proved unnecessary for this study. Thus, all children had to press the right key for means of transportation and the left key for animals. Overall, the same amount of experimental trials as in Study 1 was used in Study 2 (i.e., 20 blocks with 30 trials each).

\section{Procedure}

We conducted Study 2 during the summer holidays of 2004, reasoning that parents would be more willing to participate in a study that requires discontinuation of medication during holidays as opposed to during the school year. We tested each child in a single session lasting approximately $2 \mathrm{~h}$. In addition, we quizzed each parent(s) in an interview to make sure that the children had not received any psychostimulant medication $48 \mathrm{~h}$ prior to testing.

The procedure for this study closely mirrored the procedure used in Study 1 with the following exceptions: After the first assessment (20 blocks with 30 trials each without medication) each participant completed a final questionnaire asking them to rate three statements regarding their commitment to perform well on the task at hand: "I intended to do well on the task;" "It makes a difference for me to be good on this task;" and "It was important for me to be good on this task." Participants rated these three statements using a seven-point scale ( $1=$ strongly agree, $7=$ strongly disagree $)$.

Next, participants took a 45-min break. During this break, participants received their medication (MPH) from their parents, and thereafter watched a cartoon (The Call of the Simpsons, Groening, 1990). Subsequently, the participants repeated the experimental procedure as described above (i.e., assignment of goal versus implementation intention, 20 blocks with 30 trials each, final questionnaire).

Results

\section{Response inhibition}

Table 3 shows the results from response inhibitions in the goal and implementation intention conditions, with and without medication. A repeated measurement ANOVA was conducted to compare the response inhibition from the first experimental phase (without medication) to the response inhibition from the second experimental phase (with medication) over the intention conditions. A 2 (Intention: goal intention versus implementation intention) $\times 2$ (Medication: yes versus no) ANOVA on response inhibition revealed a marginally significant main effect of Intention, $F(1,19)=3.46$, $p=.07$, and no significant main effect of Medication. However, a significant interaction, $F(1,19)=5.78, p<.05$, between Intention and Medication emerged, indicating that the effect of forming implementation intentions was dependent on whether or not the child had taken medication. Simple effect analyses revealed no effect of intention without medication, $F(1,19)<1$, ns, but a significant effect of intention with medication, $F(1$, 19) $=5.17, p<.05$. 
Table 3 Means and standard deviations for response inhibition and classification response times in children with ADHD with and without medication (Study 2)

\begin{tabular}{lll}
\hline Medication status & Intention condition & \\
\cline { 2 - 3 } & Goal intention & Implementation intention \\
\hline Without medication & & \\
Response inhibition (\%) & $78.07(22.72)$ & $86.02(6.09)$ \\
Response time (ms) & $652.72(70.37)$ & $666.48(57.68)$ \\
With medication & $72.01(27.46)$ & $89.61(4.89)$ \\
Response inhibition (\%) & $615.72(56.88)$ & $606.19(51.28)$ \\
Response time (ms) &
\end{tabular}

Note. Standard deviations in parentheses

An analysis of covariance was used to assess whether implementation intentions still lead to better response inhibition after controlling for the age of the children. Results indicated a significant effect of Age, $F(1,19)=4.24, p=.05$, indicating that older children achieved a better stop performance. However, when controlling for age the marginally significant main effect of Intention even became significant, $F(1,19)=3.96$, $p=.05$, and the significant interaction effect between Intention and Medication stayed significant, $F(1,19)=5.55, p<.05$; again, no significant main effect of Medication was observed.

Supplementary $t$-tests showed that children with ADHD in the implementation intention condition profited from MPH. Without MPH they showed a response inhibition of $86.02 \%(S D=6.09)$ and with $\mathrm{MPH}$ their response inhibition increased to $89.61 \%(S D=4.89), t(19)=2.05, p=.06$. Children in the goal intention condition, on the other hand, did not improve their response inhibition under medication but tended to exhibit a decrease in performance (without $\mathrm{MPH}, M=78.07 \%, S D=22.72$; with MPH, $M=72.01 \%, S D=27.46 ; t(19)<1, n s)$.

Moreover, additional $t$-tests revealed that children with ADHD and without medication did not profit significantly from forming additional implementation intentions $(M=86.02, S D=6.09$ vs. $M=78.07, S D=22.72)$; $t(19)<1, n s$. For children with ADHD and medication, however, implementation intentions revealed their beneficial effects (implementation intention condition: $M=89.61, S D=4.89$; goal intention condition: $M=72.01, S D=27.64 ; t(19)=2.27, p<.05)$.

\section{Further analyses}

Response times in the classification task. Table 3 illustrates the results concerning the response times in the goal and implementation intention conditions, with and without medication. A 2 (Intention: goal intention versus implementation intention) $\times 2$ (Medication: yes versus no) repeated measurement ANOVA on response times yielded a significant main effect of Medication, $F(1,19)=19.41, p<.001$, indicating that the children generally profited from medication with MPH concerning their response times (without medication: $M=659.61 \mathrm{~ms} ; S D=64.02 \mathrm{~ms}$; with medication: $M=610.95 \mathrm{~ms}$; $S D=54.08 \mathrm{~ms}$ ). Neither the main effect of Intention, nor the interaction effect of Medication and Intention were significant.

Task commitment questionnaire. We summarized the three interview items to form one index assessing task commitment. Prior to medication, children with ADHD in the two intention conditions did not show a significant difference in their task commitment 
(Cronbach's $\alpha=.58$; goal intention without medication: $M=2.76, S D=1.11$; implementation intention without medication: $M=2.26, S D=1.19 ; t(19)<1, n s$ ); similar results were observed after medication (Cronbach's $\alpha=.65$; goal intention with medication: $\quad M=2.43, S D=1.17$; implementation intention with medication: $M=1.96, S D=.90 ; t(19)<1, n s)$. This pattern of findings indicates that the beneficial effects of implementation intentions on inhibition performance cannot be alternatively explained by an increase in commitment to do well on the task at hand.

\section{Discussion}

The marginally significant main effect of implementation intentions on response inhibition (that became significant when controlling for age) is consistent with the findings from Study 1. A meta-analysis of the implementation intention effects of Studies 1 and 2 taken together indicates that the effect size is medium $(d=0.48, N=50$, $k=2$ ), homogenous (Chi-square $(1)=0.19, n s)$, and significant $(95 \% C I=-0.08$ to 1.0 , $p=0.04)$.

Furthermore, the interaction between type of intention (goal intention versus implementation intention) and medication status (with MPH versus without MPH) indicates that the combination of the volitional strategy of forming implementation intentions with MPH medication is the most effective. This observation is in line with previous research demonstrating that children with ADHD tend to show impaired planning abilities (e.g., Clarke, Prior, \& Kinsella, 2000) that can be restored with MPH (e.g., Arnsten \& Dudley, 2005). It thus appears that MPH fosters the cognitive processes related to the formation of and acting on if-then plans (i.e., implementation intentions).

\section{General discussion}

The present research explores the effects of implementation intentions on inhibition performances in children with ADHD. Supporting the general prediction that action control benefits as a result of forming implementation intentions, children in our study with ADHD who formed implementation intentions achieved a better inhibition performance in a Go/No-Go task than ADHD participants who merely formed goal intentions. Apparently, furnishing inhibition goals with respective implementation intentions helps children with ADHD to overcome inhibition problems in a classic SST. Importantly, the beneficial response inhibition effect of implementation intentions had no costs for participants, that is, they did not display slowed response times for the Go responses. Children with ADHD and with implementation intentions as compared to those with mere goal intentions not only showed better response inhibition in the NoGo trials, but also faster (Study 1) or equally fast (Study 2) responses in the Go trials (i.e., the classification of means of transportation versus animals).

\section{Limitations of the present studies}

One limitation of the present studies is the homogeneity of the sample, e.g., that only boys participated. We decided to limit our participant pool to boys because ADHD is more prevalent in boys than in girls (Barkley, 1990). As of yet, however, the influence of gender on executive functioning in children with ADHD is unclear (Berlin, Bohlin, \& Rydell, 2003), and therefore one wonders whether implementation intentions would 
benefit both boys and girls with ADHD alike when it comes to performing executive function tasks.

Moreover, as in most other studies investigating children with ADHD, many participants in the present research had comorbid disorders. We do not consider this a limitation of the interpretation of our results as most children with ADHD have comorbid disorders (Tannock, 1998). Rather, the fact that children with ADHD with various comorbid disorders participated in our studies supports the generalizability of the present results to clinical samples (Jensen, 2003). On the other hand, it seems worthwhile to examine as many children with ADHD as needed to form subgroups of different comorbidities in future studies.

Further, control children without ADHD were not able to profit from implementation intentions which appears to be a consequence of low task difficulty for children without an action-control related disorder (response inhibition was close to $90 \%$ already in the goal intention condition). Hence, further research should analyze task performance under different levels of task difficulty. It seems very well possible that joint effectiveness of implementation intentions and MPH is most pronounced when challenging executive function tasks are to be performed.

Another limitation of the present studies is that the participating children's overall intellectual ability was not assessed. In light of recent discussions (e.g., Frazier, Demaree, \& Youngstrom, 2004) whether the weak performances on executive functions tasks observed in children with ADHD are the result of either an overall lower intellectual ability level or specific executive dysfunctions, it would have been helpful to know our research participants' general intelligence scores. This information would have also enabled us to explore whether implementation intentions help performance on the SST task for high versus low intelligence children alike.

Lastly, one limitation of Study 2 might be seen in the fact that the participating children performed the experimental task twice, at first without medication and following right afterwards with medication. Therefore, a practice effect might have contributed to participants' second performance, and not just the effect of the medication. However, as this potential practice effect should be the same in the goal intention/implementation intention condition as well as the medication/no medication condition, we only observed an interaction effect of intention condition and medication. We therefore do not consider this as a limitation to the internal validity of our Study 2 because it speaks against the assumption that implementation intentions merely intensify a practice effect. If anything, it is the external validity of the present findings that is threatened, that is, it is unclear whether the present findings on goal versus implementation intentions guided executive performances under medication versus no medication generalize to situations where children with ADHD have no practice with the executive functions task at issue.

\section{Implications for ADHD interventions}

These studies demonstrate that implementation intentions help children with ADHD to overcome problems of behavior inhibition. In addition to their theoretical significance, these findings have important applied implications. Teaching children with ADHD the strategy of forming implementation intentions would be an important supplement to existing therapy programs. Moreover, implementation intentions can be used in combination with psychostimulant medication as a strategy to obtain enhanced outcomes. 
Years ago, Meichenbaum and colleagues (e.g., Meichenbaum \& Goodman, 1971) demonstrated that impulsive children could be instructed to talk to themselves. Specifically, these researchers conducted several experiments in which they taught children to use private speech (e.g., "Good, I'm doing fine so far. Remember go slow!") in a self-instruction training. Results indicated that children committed fewer errors when they participated in this training, whereby the self-instructions commonly spelled out suitable self-monitoring or self-evaluation strategies. Thus, Meichenbaum contributed an important detail to self-regulation research in impulsive children that is in line with the present findings: children with action control difficulties (e.g., impulsive children) can profit from self-instructions. However, our studies go beyond Meichenbaum's research suggesting that self-instruction entailing the planning of an action in an "if-then" format is superior to a goal intention self-instruction. Self-instruction techniques seem to be particularly helpful when focusing on cognitive strategies of planning that automate action control as implementation intentions do.

Implications for implementation intention research

The present studies are of additional theoretical significance in the field of implementation intention research (summary by Sheeran \& Gollwitzer, 2006). First, these studies once again support findings regarding the effect of implementation intentions on performance in tasks which tax executive functions (Cohen et al., in press). Whenever high levels of executive control are required by the task at hand, if-then plans can be used to restructure the task in one's mind (by specifying critical stimuli and appropriate responses in advance), thus facilitating successful task performance. Second, the present findings are consistent with other studies demonstrating implementation intention effects in critical populations (Brandtstaetter et al., 2001; Lengfelder \& Gollwitzer, 2001). All of the clinical groups analyzed so far (e.g., drug addicts during withdrawal, hospitalized schizophrenic patients, and patients with frontal lesions) are known to have problems of action control and thus benefit from forming implementation intentions as this self-regulation strategy apparently compensates for executive dysfunctions. Third, this was the first time that implementation intentions were investigated successfully in a sample of children. Further research should examine the effects of implementation intentions in children with psychological disorders other than ADHD, for instance, psychotic youth who fail to control aggressive impulses. Finally, this was the first time that the interaction of implementation intentions and psychostimulant medication was tested. As both implementation intentions and psychostimulant medication have supportive effects on executive functions, further research should explore the neural basis of their relationship.

\section{Conclusion}

Children with ADHD can benefit from self-regulatory instructions in the form of if-then plans. This goes beyond the classic self-regulation research as our studies suggest that self-instructions in the form of implementation intentions (i.e., if-then statements) are superior to goal intention self-instructions. Further research is necessary to explore whether forming such plans can be taught to children with ADHD as a meta-cognitive strategy to be applied in everyday life. 


\section{References}

Aarts, H., \& Dijksterhuis, A. (2000). Habits as knowledge structures: Automaticity in goal-directed behavior. Journal of Personality and Social Psychology, 78, 53-63

Arbeitsgruppe Deutsche Child Behavior Checklist. (1998). Elternfragebogen über das Verhalten von Kindern und Jugendlichen. Deutsche Bearbeitung der Child Behavior Checklist (CBCL/4-18). Köln: Arbeitsgruppe Kinder-, Jugend- und Familiendiagnostik (KJFD). [German consensus version of the Child Behavior Checklist (CBCL/4-18)]

Arnsten, A. F. T., \& Dudley, A. G. (2005). Methylphenidate improves prefrontal cortical cognitive function through $\alpha 2$ adrenoceptor and dopamine D1 receptor actions: Relevance to therapeutic effects in Attention Deficit Hyperactivity Disorder. Behavioral Brain Functions, 1, 1-9.

Barkley, R. A. (1990). Attention-deficit/hyperactivity disorder: A handbook for diagnosis and treatment. New York: Guilford.

Barkley, R. A. (1997). ADHD and the nature of self-control. New York: Guilford Press.

Bedard, A., Ickowicz, A., Logan, G. D., Hogg-Johnson, S., Schachar, R., \& Tannock, R. (2003). Selective inhibition in children with attention-deficit hyperactivity disorder off and on stimulant medication. Journal of Abnormal Child Psychology, 31, 315-327.

Berlin, L., Bohlin, G., \& Rydell, A. (2003). Relations between inhibition, executive functioning, and ADHD symptoms: A longitudinal study from age 5 to 81/2 years. Child Neuropsychology, 9, 255-266.

Booth, J. R., Burman, D. D., Meyer, J. R., Lei, Z., Trommer, B. L., Davenport, N. D., Li, W., Parrish, T. B., Gitelman, D. R., \& Mesulam, M. M. (2005). Larger deficits in brain networks for response inhibition than for visual selective attention in attention deficit hyperactivity disorder. Journal of Child Psychology and Psychiatry, 46, 94-11.

Brandstaetter, V., Lengfelder, A., \& Gollwitzer, P. M. (2001). Implementation intentions and efficient action initiation. Journal of Personality and Social Psychology, 81, 946-960.

Clarke, C., Prior, M., \& Kinsella, G. (2000). Do executive function deficits differentiate between adolescents with ADHD and Oppositional Defiant/Conduct Disorder? A neuropsychological study using the Six elements test and Hayling sentence completion test. Journal of Abnormal Child Psychology, 28, 403-414.

Cohen, A., Bayer, U. C., Jaudas, A., \& Gollwitzer, P. M. (in press). Implementation intentions and executive control: Task switch performance and the Simon effect. Psychological Research.

Durston, S. (2003). A review of the biological bases of ADHD: What have we learned from imaging studies? Mental Retardation \& Developmental Disabilities Research Reviews, 9, 184-195.

Fischer, M., Barkley, R. A., Smallish, L., \& Fletcher, K. (2005). Executive functioning in hyperactive children as young adults: Attention, inhibition, response perseveration, and the impact of comorbidity. Developmental Neuropsychology, 27, 107-133.

Frazier, T. W., Demaree, H. A., \& Youngstrom, E. A. (2004). Meta-analysis of intellectual and neuropsychological test performance in attention-deficit hyperactivity disorder. Neuropsychology, $18,543-555$.

Gollwitzer, P. M. (1993). Goal achievement: The role of intentions. In W. Stroebe, \& M. Hewstone (Eds.), European review of social psychology (Vol. 4, pp. 141-185). London: Wiley.

Gollwitzer, P. M. (1996). The volitional benefits of planning. In P. M. Gollwitzer, \& J. A. Bargh (Eds.), The psychology of action: Linking cognition and motivation to behavior (pp. 287-312). New York: Guilford Press.

Gollwitzer, P. M. (1999). Implementation intentions: Strong effects of simple plans. American Psychologist, 54, 493-503.

Gollwitzer, P. M., \& Brandstaetter, V. (1997). Implementation intentions and effective goal pursuit. Journal of Personality and Social Psychology, 73, 186-199.

Gollwitzer, P. M., Bayer, U., \& McCulloch, K. (2005). The control of the unwanted. In R. Hassin, J. Uleman, \& J. A. Bargh (Eds.), The new unconscious (pp. 485-515). Oxford: Oxford University Press.

Gollwitzer, P. M., \& Sheeran, P. (2006). Implementation intentions and goal achievement: A metaanalysis of effects and processes. Advances of Experimental Social Psychology, 38, 69-119.

Groening, M. (Writer). (1990). The call of the Simpsons [Television series episode]. In J. L. Brooks, \& S. Simon (Producer), The Simpsons. Los Angeles: Twentieth Century Fox.

Halperin, J. M., \& Schulz, K. P. (2006). Revisiting the role of the prefrontal cortex in the pathophysiology of ADHD. Psychological Bulletin, 132, 560-581.

Hood, J., Baird, G., Rankin, P. M., \& Isaacs, E. (2005) Immediate effects of methylphenidate on cognitive attention skills of children with attention-deficit-hyperactivity disorder. Developmental Medicine and Child Neurology, 47, 408-414. 
Jensen, P. S. (2003). Comorbidity and child psychopathology: recommendations for the next decade. Journal of Abnormal Child Psychology, 31, 293-300.

Jensen, P. S., Hinshaw, S. P., Swanson, J. M., Greenhill, L. L., Conners, C. K., Arnold, L. E., Abikoff, H. B., Elliott, G., Hechtman, L., Hoza, B., March, J. S., Newcorn, J. H., Severe, J. B., Vitiello, B., Wells, K., \& Wigal, T. (2001). Findings from the NIMH Multimodal Treatment Study of ADHD (MTA): Implications and applications for primary care providers. Journal of Developmental \& Behavioral Pediatrics, 22, 60-73.

Lengfelder, A., \& Gollwitzer, P. M. (2001). Reflective and reflexive action control in patients with frontal brain lesions. Neuropsychology, 15, 80-100.

Logan, G. D., \& Cowan, W. B. (1984). On the ability to inhibit thought and action: A theory of an act of control. Psychological Review, 91, 295-327.

Manly, T., Anderson, V., Nimmo-Smith, I., Turner, A., Watson, P., \& Robertson, I. H. (2001). The differential assessment of children's attention: The Test of Everyday Attention for Children (TEACh), normative sample and ADHD performance. The Journal of Child Psychology and Psychiatry and Allied Disciplines, 42, 1065-1081.

Mehta, M. A., Owen, A. M., Sahakian, B. J., Mavaddat, N., Pickard, J. D., \& Robbins, T. W. (2000). Methylphenidate enhances working memory by modulating discrete fornatl and parietal lobe regions in the human brain. The Journal of Neuroscience, 20, 1-6.

Meichenbaum, D. H., \& Goodman, J. (1971). Training impulsive children to talk to themselves: A means of developing self-control. Journal of Abnormal Psychology, 77, 115-126.

Midha, K. K., McKay, G., Rawson, M. J., Korchinski, E. D., \& Hubbard, J. W. (2001). Effects of food on the pharmacokinetics of Methylphenidate. Pharmaceutical Research, 18, 1185-1189.

Miyake, A., Friedman, N. P., Emerson, M. J., Witzki, A. H., \& Howerter, A. (2000). The unity and diversity of executive functions and their contributions to complex "frontal lobe" tasks: A latent variable analysis. Cognitive Psychology, 41, 49-100.

Miyake, A., Friedman, N. P., Rettinger, D. A., Shah, P., \& Hegarty, M. (2001). How are visuospatial working memory, executive functioning, and spatial abilities related? A latent-variable analysis. Journal of Experimental Psychology: General, 130, 621-640.

Nichols, S. L., \& Waschbusch, D. A. (2004). A review of the validity of laboratory cognitive tasks used to asses symptoms of ADHD. Child Psychiatry \& Human Development, 34, 297-315.

Oosterlaan, J., Logan, G. D., \& Sergeant, J. A. (1998). Response inhibition in ADHD, CD, comorbid ADHD + CD, anxious and normal children: a meta-analysis of studies with the stop task. Journal of Child Psychology and Psychiatry, 39, 411-425.

Oosterlaan, J., \& Sergeant, J. A. (1996). Inhibition in ADHD, aggressive, and anxious children: A biologically based model of child psychopathology. Journal of Abnormal Child Psychology, 24, 1936.

Overtoom, C. C., Kenemans, J. L., Verbaten, M. N., Kemner, C., van der Molen, M. W., van Engeland, H., Buitelaar, J. K., \& Koelega, H. S. (2002). Inhibition in children with attention-deficit/ hyperactivity disorder: A psychophysiological study of the stop task. Biological Psychiatry, 51, 668676.

Orbell, S., Hodgkins, S., \& Sheeran, P. (1997). Implementation intentions and the theory of planned behavior. Personality and Social Psychology Bulletin, 23, 945-954.

Owens, J. S., \& Hoza, B. (2003). The role of inattention and hyperactivity/impulsivity in the positive illusory bias. Journal of Consulting and Clinical Psychology, 71, 680-691.

Parks-Stamm, E., Gollwitzer, P. M., \& Oettingen, G. (2007). Action control by implementation intentions: Effective cue detection and efficient response initiation. Social Cognition, 25, 248-266.

Pennington, B. F., Bennetto, L., McAleer, O., \& Roberts, R. J. (1996). Executive functions and working memory: Theoretical and measurement issues. In G. R. Lyon, \& N. A. Krasnegor (Eds.), Attention, memory, and executive function (pp. 327-348). Baltimore, MD, US: Paul H. Brookes Publishing Co.

Pliszka, S. R., Glahn, D. C., Semrud-Clikeman, M., Franklin, C., Perez, R., Xiong, J., \& Liotti, M. (2006). Neuroimaging of inhibitory control areas in children with ADHD who were treatment naive or in long-term treatment. American Journal of Psychiatry, 163, 1052-1060.

Pliszka, S. R., Liotti, M., \& Woldorff, M. G. (2000). Inhibitory control in children with attention-deficit/ hyperactivity disorder: event-related potential identify the processing component and timing of an impaired right-frontal response-inhibition mechanism. Biological Psychiatry, 48, 238-246.

Pliszka, S. R., Borcherding, S. H., Spratley, K., Leon, S., \& Irick S. (1997). Measuring inhibitory control in children. Developmental and Behavioral Pediatrics, 18, 254-259.

Posner, M. L, \& Rothbart, M. K. (2000). Developing mechanisms of self-regulation. Development \& Psychpathology, 12, 427-441. 
Reid, R., Trout, A. L., \& Schartz, M. (2005). Self-regulation interventions for children with ADHD. Exceptional Children, 71, 361-377.

Rosvold, H. E., Mirsky, A. F., Sarason, I., Bransome, E. D., \& Beck, L. H. (1956). A continuous performance test of brain damage. Journal of Consulting Psychology, 20, 343-350.

Scheres, A., Oosterlaan, J., Geurts, H., Morein-Zamir, S., Meiran, N., Schut, H., Vlasveld, L., \& Sergeant, J. A. (2004). Executive functioning in boys with ADHD: Primarily an inhibition deficit? Archives of Clinical Neuropsychology, 19, 569-594.

Seijts, G. H., \& Latham, G. P. (2000). The construct of goal-commitment: Measurement and relationships with task performance. In R. Goffin, \& E. Helmes (Eds.), Problems and solutions in human assessment (pp. 315-332). Dordrecht: Kluwer Academic.

Sheeran, P., \& Orbell, S. (1999). Implementation intentions and repeated behavior: Augmenting the predictive validity of the theory of planned behavior. European Journal of Social Psychology, 29, 349-369.

Sheeran, P., Webb, T. L., \& Gollwitzer, P. M. (2005). The interplay between goal intentions and implementation intentions. Personality and Social Psychology Bulletin, 31, 87-98.

Smith, A., \& Taylor, E. (2006). Response inhibition and hyperactivity in clinical, non-clinical populations: A meta-analysis using the stop task. In R. D. Oades (Ed.), AD/HD and HKS: Current ideas and ways forward (pp. 203-225). Hauppauge: Nova Science Publishers.

Smith, A., Taylor, E., Brammer, M., Toone, B., \& Rubia, K. (2006). Task-specific hypoactivation in prefrontal and temporoparietal brain regions during motor inhibition and task switching in medication-naïve children and adolescents with ADHD. American Journal of Psychiatry, 163, 10441051.

Sonuga-Barke, E. J. S. (2002). Psychological heterogeneity in AD/HD-a dual pathway model of behavior and cognition. Behavioral Brain Research, 130, 29-36.

Tannock, R. (1998). Attention deficit hyperactivity disorder: advances in cognitive, neurobiological, and genetic research. Journal of Child Psychology and Psychiatry, 39, 65-99.

The World Medical Association (2004). World Medical Association declaration of Helsinki: Ethical principles for medical research involving human subjects. Retrieved June 3, 2006 from http:// www.wma.net/e/policy/b3.htm.

Webb, T. L., \& Sheeran, P. (2003). Can implementation intentions help to overcome ego-depletion? Journal of Experimental Social Psychology, 39, 279-286.

Webb, T. L., \& Sheeran, P. (2007). How do implementation intentions promote goal attainment? A test of component processes. Journal of Experimental Social Psychology, 43, 295-302.

WHO (1991). Internationale Klassifikation psychischer Stoerungen: ICD 10, Kapitel V. Bern: Huber. [Tenth revision of the International classification of diseases, Chapter V (F): Mental and behavioral disorders].

Willcutt, E. G., Pennington, B. F., Olson, R. K., Chhabildas, N., \& Hulslander, J. (2005). Neuropsychological analyses of comorbidity between reading disability and attention deficit hyperactivity disorder: In search of the common deficit. Developmental Neuropsychology, 27, 35-78.

Wu, K. K., Anderson, V., \& Castiello, U. (2006). ADHD and working memory: A task switching paradigm. Journal of Clinical and Experimental Neuropsychology, 28, 1288-1306.

Zelazo, P. D., Qu, L., \& Müller, U. (2005). Hot and cool aspects of executive function: Relations in early development. In W. Schneider, R. Schumann-Hengsteler, \& B. Sodian (Eds.), Young children's cognitive development: Interrelationships among executive functioning, working memory, verbal ability, and theory of mind (pp. 71-93). Mahwah, NJ, US: Lawrence Erlbaum Associates, Publishers.

Zhu, J. (2004). Locating volition. Consciousness and Cognition, 13, 302-322. 